\title{
Perfil metabólico e antropométrico dos pacientes obesos e não obesos portadores de esteatose hepática não alcoólica
}

\author{
Metabolic and anthropometric profile of obese and non-obese patients with non-alcoholic \\ liver steatosis
}

\author{
Perfil metabólico y antropométrico de pacientes obesos y no obesos con esteatosis \\ hepática no alcohólica
}

\begin{abstract}
Jéssica Teles Santana ${ }^{1 *}$, Allan Victor Hora Mota ${ }^{1}$, Yasmin Hora Góis Gonzaga1 ${ }^{1}$, Raphaella Maria Oliveira Pereira Gomes ${ }^{1}$, Lívia Carvalho Melo ${ }^{1}$, Victor Fernando Costa Macedo Noronha ${ }^{1}$, Anny Carolyne Oliveira Lima Santos ${ }^{1}$, Joseane Barbosa de Jesus², Sonia Oliveira Lima ${ }^{1}$, Josilda Ferreira Cruz ${ }^{1}$.
\end{abstract}

\begin{abstract}
RESUMO
Objetivo: Avaliar o perfil metabólico e antropométrico dos pacientes obesos e não obesos portadores de esteatose hepática não alcoólica diagnosticados pela ultrassonografia abdominal. Métodos: Estudo clínico e prospectivo realizado no período entre abril de 2019 a fevereiro de 2020 em um centro de referência em ultrassonografia em Aracaju - SE. Foram avaliados índice de massa corporal, circunferência abdominal e dosados glicemia de jejum, colesterol total, lipoproteínas de alta densidade, lipoproteínas de baixa densidade, lipoproteínas de muito baixa densidade e triglicerídeos de todos os pacientes. Resultados: Foram estudados 85 pacientes portadores de esteatose hepática (45 não obesos e 40 obesos). Deles, 43 pacientes corresponderam ao grau leve e 42 ao grau moderado/severo. Com relação as condições clinicas, 78 pacientes apresentaram dislipidemia, 13 tinham diabetes mellitus tipo 2, 27 tinham hipertensão arterial sistêmica, 40 apresentaram síndrome metabólica e 65 pacientes foram portadores de obesidade central. Houve associação estatisticamente significativa no grupo dos pacientes obesos para triglicerídeos e circunferência abdominal em relação a gravidade da infiltração gordurosa. Entretanto, não houve esta associação no grupo dos pacientes não obesos. Conclusão: A obesidade e as suas condições metabólicas são fatores de risco para a gravidade da esteatose hepática não alcóolica.
\end{abstract}

Palavras-chave: Fígado gorduroso, Obesidade, Ultrassonografia.

\begin{abstract}
Objective: To evaluate the metabolic and anthropometric profile of obese and non-obese patients with nonalcoholic hepatic steatosis diagnosed by abdominal ultrasound. Methods: Clinical and prospective study carried out between April 2019 and February 2020, at a reference center for ultrasonography in Aracaju-SE. Body mass index, waist circumference and fasting blood glucose levels, total cholesterol, high-density lipoproteins, low-density lipoproteins, very low-density lipoproteins and triglycerides of all patients were evaluated. Results: 85 patients with hepatic steatosis ( 45 non-obese and 40 obese) were studied. Of these, 43 patients corresponded to mild and 42 to moderate / severe. Regarding clinical conditions, 78 patients had dyslipidemia, 13 had type 2 diabetes mellitus, 27 had systemic arterial hypertension, 40 had metabolic syndrome and 65 patients had central obesity. There was a statistically significant association in the group of obese patients for triglycerides and waist circumference in relation to the severity of fatty infiltration. However, there was no such association in the group of non-obese patients. Conclusion: Obesity and its metabolic conditions are risk factors for the severity of non-alcoholic liver steatosis.
\end{abstract}

Keywords: Fatty liver, Obesity, Ultrasonography.

1 Universidade Tiradentes (UNIT), Aracaju - SE. * E-mail: jessicateles08@gmail.com

2 Universidade Federal de Sergipe (UFS), Aracaju - SE. 


\section{RESUMEN}

Objetivo: Evaluar el perfil metabólico y antropométrico de pacientes obesos y no obesos con esteatosis hepática no alcohólica diagnosticada por ecografía abdominal. Métodos: Estudio clínico y prospectivo realizado entre abril de 2019 y febrero de 2020, en un centro de referencia en ecografía en Aracaju-SE. Se evaluó el índice de masa corporal, circunferencia de cintura y niveles de glucosa en sangre en ayunas, colesterol total, lipoproteínas de alta densidad, lipoproteínas de baja densidad, lipoproteínas de muy baja densidad y triglicéridos de todos los pacientes. Resultados: Se estudiaron 85 pacientes con esteatosis hepática (45 no obesos y 40 obesos). De estos, 43 pacientes correspondieron a leves y 42 a moderados / graves. En cuanto a las condiciones clínicas, 78 pacientes tenían dislipidemia, 13 tenían diabetes mellitus tipo 2, 27 tenían hipertensión arterial, 40 tenían síndrome metabólico y 65 pacientes tenían obesidad central. Hubo una asociación estadísticamente significativa en el grupo de pacientes obesos para los triglicéridos y la circunferencia de la cintura en relación con la gravedad de la infiltración grasa. Sin embargo, no hubo tal asociación en el grupo de pacientes no obesos. Conclusión: La obesidad y sus condiciones metabólicas son factores de riesgo para la gravedad de la esteatosis hepática no alcohólica.

Palabras clave: Hígado graso, Obesidad, Ultrasonido.

\section{INTRODUÇÃO}

A doença hepática gordurosa não alcoólica (DHGNA) é a afecção do fígado mais prevalente em todo o mundo afetando $20-25 \%$ da população adulta. Em $25 \%$ dos pacientes, a DHGNA progride para esteatohepatite não alcoólica (EHNA) o que aumenta o risco de desenvolvimento de cirrose, insuficiência hepática e carcinoma hepatocelular (SCHWABE RF, et al., 2020). Portanto, a prevenção e o tratamento da DHGNA são altamente relevantes (PINTÓ X, et al., 2019).

A DHGNA caracteriza-se pela deposição de lipídios no parênquima hepático excedendo $5 \%$ do peso do órgão na ausência de etiologias virais, alcoólicas ou metabólicas sendo uma patologia que acomete de $73 \%$ a $90 \%$ dos pacientes obesos (COTRIM HP, et al., 2016; BAGHERI LK, et al., 2013). Considerando a atual epidemia da obesidade desde a infância, o aumento do diabetes mellitus tipo 2 (DM2) e de outros fatores, estima-se que a prevalência da DHGNA nos indivíduos continue a aumentar. Isso terá um impacto importante na saúde pública refletindo nos custos de saúde, incluindo a necessidade de transplante hepático para o qual a EHNA já está próxima de se tornar a indicação mais comum (COTTER TG e RINELLA M, 2020).

A obesidade é uma doença de natureza multifatorial resultante de fatores genéticos, epigenéticos, fisiológicos, comportamentais, socioculturais e ambientais que levam a um desequilíbrio entre o consumo e gasto energético durante um período prolongado (BRAY GA, et al., 2016). É uma patologia crônica definida por um Índice de Massa Corporal (IMC) $\geq 30 \mathrm{~kg} / \mathrm{m}^{2}$ e é um fenótipo clínico comum associado tanto a DHGNA como a síndrome metabólica (SM) (KIM D e KIM WR, 2016).

Evidências crescentes mostraram que a taxa de prevalência de DHGNA nas populações não obesas não é incomum. Globalmente, a prevalência da patologia no paciente não obeso varia amplamente de 3\% a $30 \%$. A DHGNA pode representar um grupo de condições em que vários processos patogenéticos podem estar presentes e serem diferentes entre pacientes obesos e não obesos, apesar de condições clínicas e apresentações histopatológicas similares (KIM D e KIM WR, 2016).

A patogênese da DHGNA no não obeso pode estar relacionada à predisposição genética que leva ao acúmulo de triglicerídeos (TG) intra-hepático e à resistência à insulina, a componentes da SM, a fatores dietéticos e, principalmente, ao acúmulo de obesidade visceral que parece ser um fator etiológico importante para a presença de DHGNA nesse grupo (YOUSEF MH, et al., 2017).

No tocante à fisiopatologia, a deposição excessiva de gordura no fígado pode ocorrer devido a um aumento na liberação de ácidos graxos ( $A G)$ pelo tecido adiposo, um aumento na síntese de um novo ácido graxo através da lipogênese pelo excesso de carboidratos, um aumento na gordura da dieta, uma diminuição na betaoxidação dos AG livres, diminuição na exportação de partículas de lipoproteínas de muito baixa densidade (VLDL) ou a combinação desses fatores. Em condições normais, o fígado não serve como depósito de gordura e a concentração de TG hepáticos é baixa. No entanto, há um fluxo considerável de TG e AG dentro e fora do fígado em resposta a alimentos e jejum (KAWANO Y e COHEN DE, 2013; RESS C e KASER S, 2016). 
Para o diagnóstico de DHGNA, a biópsia hepática continua sendo o exame mais específico para avaliar a natureza e a gravidade da infiltração gordurosa no fígado. No entanto, seu custo e o potencial de complicações levaram a um interesse considerável no desenvolvimento de novos métodos não invasivos para a prática clínica. A ultrassonografia (USG) é um método diagnóstico amplamente disponível, pouco oneroso, sem radiação ionizante possuindo uma sensibilidade de $85 \%$ e especificidade de $94 \%$ (STEFAN N, et al., 2019).

Em relação a tomografia computadorizada (TC), a TC tem uma sensibilidade de $82 \%$ e uma especificidade de $100 \%$ quando o conteúdo de gordura é $\geq 30 \%$, porém apresenta alto custo. A ressonância magnética (RM) é o método de imagem mais sensível para detectar aumento de gordura intra-hepática e pode detectar esteatose com apenas 3\% de teor de gordura. Entretanto, esse método possuí desvantagens como 0 alto custo também e pouca disponibilidade nos serviços de saúde (GRAFFIGNA M, et al., 2017).

O objetivo do presente estudo foi avaliar os índices antropométricos e metabólicos dos pacientes obesos e não obesos portadores da estaetose hepática não alcoólica diagnosticada pela USG a fim de permitir a instituição precoce de um tratamento multidisciplinar com intuito de modificar o curso natural da doença e as suas complicações diminuindo a mortalidade desses pacientes.

\section{MÉTODOS}

Trata-se de um estudo clínico, transversal, descritivo, com abordagem analítica quantitativa e qualitativa. Os dados foram coletados em um centro de referência de USG no município de Aracaju - SE, Brasil, no período entre Abril/2019 a Fevereiro/2020 após aprovação do Comitê de Ética em Pesquisa da Universidade Tiradentes sob número de parecer 3.238.258. Os exames foram realizados por um único examinador, sendo este um médico com experiência no diagnóstico de esteatose hepática não alcoólica, com o mesmo aparelho de USG e boa resolução de imagem.

Os critérios de inclusão foram adultos de ambos os sexos na faixa etária de 18 a 70 anos e portadores de esteatose hepática não alcóolica na USG. Os critérios de exclusão foram: pacientes com consumo de álcool $\geq 140 \mathrm{~g} / \mathrm{semana}$ nos homens e $\geq 70 \mathrm{~g} / \mathrm{semana}$ nas mulheres, portadores de neoplasias malignas primárias do fígado, portadores de doenças crônicas do fígado, portadores de deficiência cognitiva, pacientes em uso regular de drogas indutoras de esteatose hepática como corticosteroides, tamoxifeno e amiodarona.

As seguintes variáveis foram analisadas: IMC, circunferência abdominal, glicemia de jejum, colesterol total, lipoproteínas de alta densidade (HDL), lipoproteínas de baixa densidade (LDL), VLDL e TG em relação a variável esteatose hepática não alcoólica. O procedimento de coleta dividiu-se em quatro etapas: 01, 02, 03 e 04.

$\mathrm{Na}$ etapa 01, os pacientes, após assinatura do termo de consentimento livre e esclarecido (TCLE), responderam o questionário com informações sociodemográficas, sobre comorbidades e uso de medicamentos. No referido questionário constou informações sobre as seguintes variáveis: nome, idade, sexo, data de nascimento, naturalidade, escolaridade, profissão, frequência de etilismo, deficiência cognitiva, comorbidades associadas como hepatopatias prévias e neoplasias malignas e uso de medicamentos.

Para formular a hipótese diagnóstica da DHGNA, o consumo de álcool semanal foi considerado < $140 \mathrm{~g} /$ semana para homens e $<70 \mathrm{~g} /$ semana para mulheres, através do questionário sociodemográfico com informações sobre hábitos etílicos. A presença e frequência de etilismo foi conhecida e o consumo foi calculado utilizando-se a fórmula: dose em $\mathrm{ml} \times$ grau $\times 0,8 / 100$, onde os graus ou teor alcoólico das bebidas são conhecidos, como cerveja 4 , vinho 12 , conhaque 40 , rum 40, uísque 43 , pinga 46 (MINCIS M e MINCIS $\mathrm{R}, 2011)$.

$\mathrm{Na}$ etapa 02, os pacientes tiveram suas medidas antropométricas e pressão arterial aferidas. A avaliação antropométrica consistiu na aferição de peso e altura para realização do cálculo do IMC e mensuração da circunferência abdominal. Para a quantificação do peso corpóreo, utilizou-se uma balança portátil marca TECHLINE onde o paciente foi posto no centro da mesma em pé e descalço. Já para a realização da aferição da altura, utilizou-se um estadiômetro portátil da marca FILIZOLA com a leitura realizada no milímetro (mm) mais próximo. Os dados de peso e altura foram utilizados para o cálculo do IMC, que foi calculado pelo índice de Quetelet: razão entre o peso corpóreo em quilograma e altura em metro ao quadrado (WHO, 1995). 
O IMC foi classificado segundo critério da Organização Mundial de Saúde (OMS) em: até $24,9 \mathrm{~kg} / \mathrm{m}^{2}$ normal, de 25 a $29,9 \mathrm{~kg} / \mathrm{m}^{2}$ sobrepeso, de 30 a $34,9 \mathrm{~kg} / \mathrm{m}^{2}$ obesidade grau I ou leve, de 35 a $39,9 \mathrm{~kg} / \mathrm{m}^{2}$ obesidade grau II ou moderada e $\geq 40 \mathrm{~kg} / \mathrm{m}^{2}$ obesidade grau III ou grave. A circunferência abdominal foi medida com o paciente em pé, utilizando uma fita métrica inelástica a nível do ponto médio entre a última costela e a crista ilíaca (LOHMAN TG, et al., 1988).

Em relação a aferição da pressão arterial, foi-se utilizado um esfigmonamômetro da marca P.A. MED com um o estetoscópio da marca Littmann e realizou-se com o paciente em posição sentada. Para avaliação dos valores da pressão arterial sistêmica encontrados, utilizou-se o critério para diagnóstico de SM segundo o National Cholesterol Education Program's Adult Treatment Panel III (NCEP-ATP III), sendo considerados alterados os valores de pressão arterial $\geq 130 \mathrm{mmHg}$ ou $\geq 85 \mathrm{mmHg}$.

$\mathrm{Na}$ etapa 03, foram realizados os exames de USG. Foi utilizado aparelho de ultrassom que possuí boa resolução de imagem com transdutor convexo, dinâmico, de frequência de $3,75 \mathrm{MHZ}$. Todos os pacientes foram aconselhados acerca de um preparo adequado, ou seja, jejum de no mínimo 6 horas e uso de antiflatulento.

Quando a esteatose hepática foi encontrada, classificou-se pela USG nos graus $0,1,2$ ou 3 (SAADEH SY, et al., 2002): Grau 0: Ecogenicidade normal; Grau 1: Esteatose leve, com visualização de ecos finos do parênquima hepático, visualização normal do diafragma e de vasos intra-hepáticos; Grau 2: Esteatose moderada, com aumento difuso nos ecos finos, visualização prejudicada dos vasos intra-hepáticos e diafragma; Grau 3: Esteatose severa, com aumento importante dos ecos finos, com visualização prejudicada ou ausente dos vasos intra-hepáticos.

$\mathrm{Na}$ etapa 04, após a realização da USG abdominal, o paciente foi orientado sobre a realização dos exames bioquímicos como glicemia de jejum, colesterol total, HDL, LDL, VLDL e TG. A coleta de sangue foi realizada com jejum de 12 horas. A metodologia utilizada para a dosagem da glicose foi a oxidase, utilizando-se 0 reagente da ABBOTT Laboratórios na qual a amostra foi coletada com técnicas de punção venosa padrão em tubos com gel separador.

Em relação aos TG, as amostras foram coletadas também com tubos de gel separador e utilizada a metodologia glycerol fosfato oxidase. Nesse tipo de reação, os TG são hidrolisados enzimaticamente pela lipase formando ácidos graxos livres e glycerol. O HDL foi dosado utilizando-se um método homogêneo sem etapas de centrifugação.

Complexos antígeno-anticorpos foram formados com as lipoproteínas LDL, VLDL e quilomícrons de maneira que somente o HDL foi determinado seletivamente através de uma reação enzimática. Para a sua dosagem, foram utilizadas amostras coletadas em tubos com gel separador. Consideraram-se valores alterados: glicemia de jejum $\geq 110 \mathrm{mg} / \mathrm{dl}, \mathrm{HDL}<50 \mathrm{mg} / \mathrm{dl}$ nas mulheres e $<40 \mathrm{mg} / \mathrm{dl}$ nos homens e TG $\geq$ $150 \mathrm{mg} / \mathrm{dl}$ seguindo os critérios do NCEP-ATP III para diagnóstico de SM.

$\mathrm{Na}$ dosagem do colesterol total, a metodologia utilizada foi a enzimática onde os ésteres do colesterol foram hidrolisados enzimaticamente pela enzima colesterol esterase em colesterol e AG livres. Utilizou-se o reagente $A B B O T T$ e a amostra coletada em tubos com gel separador. Foi considerado alterado o colesterol total $>200 \mathrm{mg} / \mathrm{dl}$, seguindo os critérios da V Diretriz Brasileira de Dislipidemias e Prevenção da Aterosclerose (XAVIER HT, et al., 2013).

Os dados foram compilados no programa Microsoft Office Excel. Foram obtidas variáveis qualitativas e quantitativas. Para as variáveis qualitativas, a análise descritiva procedeu com a categorização dos dados, obtenção das respectivas frequências e percentuais e o cálculo da média, mediana, desvio padrão, mínimo e máximo foi realizado para as variáveis quantitativas. Para verificar a relação entre as variáveis qualitativas e os grupos (obesos e não obesos) foi utilizado o teste Qui-quadrado (X2) (PEARSON K, 1992).

Para o cruzamento entre as variáveis quantitativas (idade, peso, altura, IMC, circunferência abdominal, glicemia de jejum, colesterol total, LDL, HDL, TG, VLDL) foi verificado inicialmente a normalidade das distribuições dessas variáveis através do teste de Shapiro-Wilk (SHAPIRO SS e WILK M, 1965). Apenas a variável colesterol total apresentou normalidade para o cruzamento dessa variável, adotando-se assim os testes paramétricos de t-Student (1908). 
Já as demais variáveis (idade, peso, altura, IMC, circunferência abdominal, glicemia, LDL, HDL, TG, VLDL), como não foi observado normalidade, adotou-se testes não paramétricos para a análise. Então, para o cruzamento destas variáveis quantitativas com os grupos foi utilizado o teste de Mann-Whitney (MANN HB e WHITNEY DR, 1947).

O método mais adequado para o ajuste da obesidade através de modelos de regressão foi o Modelo Linear Generalizado, assumindo a variável resposta com distribuição Binomial com função de ligação logit. Esse modelo é popularmente conhecido como Regressão Logística (COX DR, 1958).

Foram gerados modelos de regressão assumindo como variáveis dependentes apenas aquelas que foram significativas na análise inferencial realizada anteriormente (circunferência abdominal, idade, SM). Os dados foram analisados através no software R, versão 3.6.1 (THE R CORE TEAM, 2019) para Windows e o nível de significância utilizado foi $p<0,05$.

\section{RESULTADOS}

Foram estudados 85 pacientes com idade média de $46,4( \pm 8,7)$ anos, variando de 24 a 59 anos e o IMC médio de $30,7( \pm 5,7) \mathrm{kg} / \mathrm{m}^{2}$, variou de 21,2 a $48,5 \mathrm{~kg} / \mathrm{m}^{2}$. Já a circunferência abdominal variou de 78 a $137 \mathrm{~cm}$ apresentando uma média de $100,2( \pm 12,9) \mathrm{cm}$. A maioria dos pacientes foi do sexo feminino $(\mathrm{n}=62 ; 72,9 \%)$.

Os pacientes foram pareados quanto ao IMC e divididos em dois grupos, obesos $(n=40)$ e não obesos $(n=45)$. Com relação as condições clinicas, verificou-se que $91,8 \%(n=78)$ dos pacientes apresentaram dislipidemia, 15,3\% $(n=13)$ tinham DM2, 31,8\% ( $n=27)$ tinham hipertensão arterial sistêmica (HAS) e 47,1\% $(\mathrm{n}=40)$ apresentaram SM (Tabela 1).

Tabela 1 - Frequência absoluta e relativa das variáveis categóricas dos pacientes portadores de esteatose hepática não alcóolica.

\begin{tabular}{lcc}
\hline & Frequência & Percentual \\
\hline Sexo & & \\
\hline Masculino & 23 & 27.1 \\
Feminino & 62 & 72.9 \\
\hline Obesidade & & \\
\hline Sim & 40 & 47.1 \\
Não & 45 & 52.9 \\
\hline Obesidade abdominal & & \\
\hline Sim & 65 & 76.5 \\
Não & 20 & 23.5 \\
\hline Dislipidemia & & \\
\hline Sim & 78 & 91.8 \\
Não & 7 & 8.2 \\
\hline Diabetes mellitus tipo 2 & & 15.3 \\
\hline Sim & 13 & 84.7 \\
Não & 72 & \\
\hline Hipertensão arterial & & 31.8 \\
Sistêmica & & 68.2 \\
\hline Sim & 27 & \\
Não & 58 & 47.1 \\
\hline Síndrome metabólica & & 52.9 \\
\hline Sim & 40 & \\
Não & 45 & \\
\hline
\end{tabular}

Fonte: Santana JT, et al., 2020.

A média de idade foi significantemente menor entre os obesos portadores de esteatose hepática não alcóolica $(43,6 \pm 9,2$ anos) do que entre os pacientes não obesos $(48,9 \pm 7,6$ anos) $(p=0,008)$ portadores dessa doença. Não houve associação estatisticamente significativa quanto ao sexo e a obesidade $(p=0,565)$. Constata-se que entre os dados antropométricos, houve associação significativa quanto a circunferência abdominal e a obesidade $(p<0,05)$ (Tabela 2$)$. 
Tabela 2 - Associação idade, dados antropométricos e sexo com presença de obesidade nos pacientes portadores de esteatose hepática não alcoólica.

\begin{tabular}{|c|c|c|c|c|c|c|c|c|c|}
\hline \multirow{3}{*}{ Variáveis } & \multicolumn{8}{|c|}{ Obesidade (IMC) } & \multirow{3}{*}{ p-valo } \\
\hline & \multicolumn{4}{|c|}{ Sim } & \multicolumn{4}{|c|}{ Não } & \\
\hline & Média & DP & Mínima & Máxima & Média & DP & Mínima & Máxima & \\
\hline Idade & 43.6 & 9.2 & 24.0 & 59.0 & 48.9 & 7.6 & 28.0 & 59.0 & 0.008 \\
\hline IMC & 35.1 & 5.1 & 30.2 & 48.5 & 26.7 & 2.3 & 21.2 & 29.9 & \\
\hline \multirow[t]{2}{*}{ Circunferência abdominal } & 109.6 & 11.9 & 90.0 & 137.0 & 91.9 & 6.8 & 78.0 & 104.0 & 0.000 \\
\hline & & $\mathbf{N}$ & $\%$ & & & $\mathbf{N}$ & $\%$ & & p-valor \\
\hline \multicolumn{10}{|l|}{ Sexo } \\
\hline Masculino & & 12 & 30 & & & 11 & 24,4 & & 0,565 \\
\hline Feminino & & 28 & 70 & & & 34 & 75,6 & & \\
\hline
\end{tabular}

Legenda: DP = Desvio Padrão; P- significância estatística.

Fonte: Santana JT, et al., 2020.

Observa-se que entre os dados clínicos, apenas houve associação estatisticamente significativa quanto a SM e a obesidade. Dos 40 pacientes que possuem a SM, 67,5\% são obesos $(p<0,05)$. Quando avaliado o grau de esteatose hepática não alcoólica, 43 pacientes foram classificados com grau leve $(50,6 \%)$ e 42 com grau moderado/severo $(49,4 \%)$ (Tabela 3 ).

Tabela 3 - Associação dos dados clínicos em relação a obesidade dos pacientes portadores de esteatose hepática não alcoólica.

\begin{tabular}{lcccccc} 
& \multicolumn{7}{c}{ Variáveis } & \multicolumn{2}{c}{ Obesidade (IMC) } & \\
\cline { 2 - 6 } & \multicolumn{2}{c}{ Sim } & Não & p- \\
Dislipidemia & N & $\%$ & N & $\%$ & valor \\
\hline Sim & 39 & 97,5 & 39 & 86,7 & 0,114 \\
Não & 1 & 2,5 & 6 & 13,3 & \\
\hline
\end{tabular}

Diabetes mellitus tipo 2

\begin{tabular}{lccccc}
\hline Sim & 8 & 20 & 5 & 11,1 & 0,256 \\
Não & 32 & 80 & 40 & 88,9 & \\
\hline
\end{tabular}

\begin{tabular}{llllll}
\hline Hipertensão arterial sistêmica & & & & & \\
\hline Sim & 15 & 37,5 & 12 & 26,7 & 0,284 \\
Não & 25 & 62,5 & 33 & 73,3 & \\
\hline
\end{tabular}

Síndrome metabólica

\begin{tabular}{llllll}
\hline Sim & 27 & 67,5 & 13 & 28,9 & $\mathbf{0 , 0 0 0}$ \\
Não & 13 & 32,5 & 32 & 71,1 & \\
\hline Grau da doença & & & & & \\
\hline Grau leve & 22 & 55 & 25 & 55,6 & 0,331 \\
Grau moderado/severo & 18 & 45 & 20 & 44,4 & \\
\hline
\end{tabular}

Legenda: DP = Desvio Padrão; P- significância estatística.

Fonte: Santana JT, et al., 2020.

Os exames bioquímicos realizados nos dois grupos de pacientes tiveram seus valores comparados. Assim, foi visto que os valores mais altos pertenceram ao grupo dos pacientes obesos. Entretanto, nenhum exame bioquímico apresentou significância estatística com a obesidade (Tabela 4). 
Tabela 4 - Relação dos exames bioquímicos em relação a obesidade dos pacientes portadores de esteatose hepática não alcoólica.

\begin{tabular}{|c|c|c|c|c|c|c|c|c|c|c|c|}
\hline \multirow{3}{*}{ Variável } & \multicolumn{10}{|c|}{ Obesidade (IMC) } & \multirow{3}{*}{$p$-valor } \\
\hline & \multicolumn{5}{|c|}{ Sim } & \multicolumn{5}{|c|}{ Não } & \\
\hline & $\mathrm{N}$ & Média & DP & Mínimo & Máximo & $\mathrm{N}$ & Média & DP & Mínimo & Máximo & \\
\hline Glicemia de jejum & 40.0 & 107.4 & 41.7 & 80.0 & 297.0 & 45.0 & 103.2 & 43.5 & 71.0 & 317.0 & 0.211 \\
\hline Colesterol Total & 40.0 & 218.3 & 45.6 & 143.0 & 362.0 & 45.0 & 212.2 & 38.1 & 121.0 & 293.0 & 0.510 \\
\hline LDL & 39.0 & 141.4 & 56.3 & 71.0 & 400.0 & 42.0 & 132.9 & 28.0 & 71.0 & 202.0 & 0.734 \\
\hline HDL & 40.0 & 43.1 & 8.6 & 28.0 & 79.0 & 45.0 & 45.3 & 10.4 & 29.0 & 68.0 & 0.282 \\
\hline Triglicerídeos & 40.0 & 215.6 & 192.0 & 79.0 & 1154.0 & 45.0 & 171.2 & 106.0 & 62.0 & 547.0 & 0.194 \\
\hline VLDL & 40.0 & 43.1 & 38.4 & 15.8 & 230.8 & 45.0 & 34.2 & 21.2 & 12.4 & 109.4 & 0.194 \\
\hline
\end{tabular}

Legenda: DP = Desvio Padrão; P- significância estatística.

Fonte: Santana JT, et al., 2020.

Quando analisados separadamente os grupos dos pacientes obesos e não obesos, apenas TG e circunferência abdominal foram significativas $(p=0,05)$ nos obesos com área sob curva ROC (do inglês, Receiver Operating Characteristic Curve) $>0.7$ para TG e circunferência abdominal em associação a gravidade da doença (Gráfico 1). Com relação aos pacientes não obesos, nenhuma das variáveis mostrou significância estatística, entretanto a glicemia evidenciou uma área $>0.7$ sob a curva ROC em comparação aos graus da infiltração gordurosa hepática (Gráfico 2).

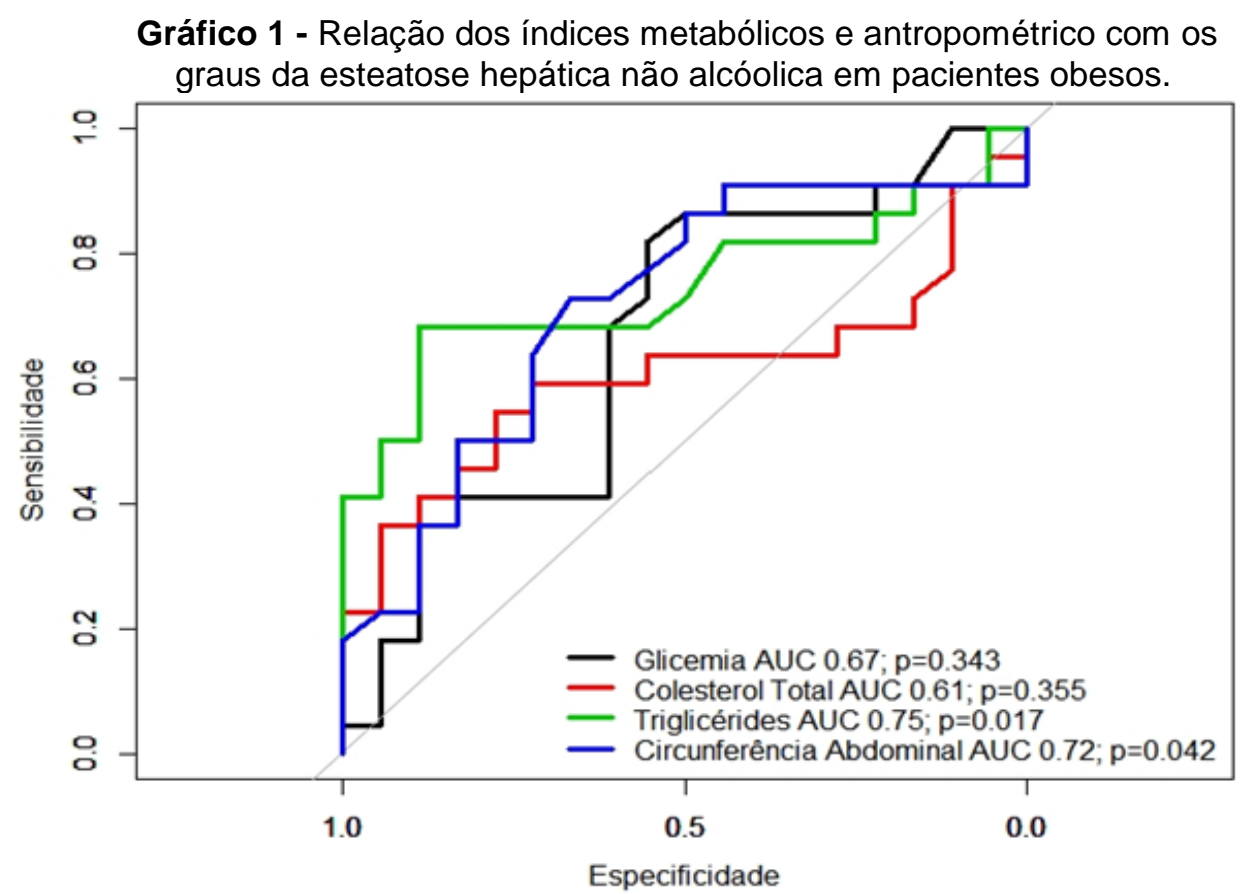

Fonte: Santana JT, et al., 2020. 


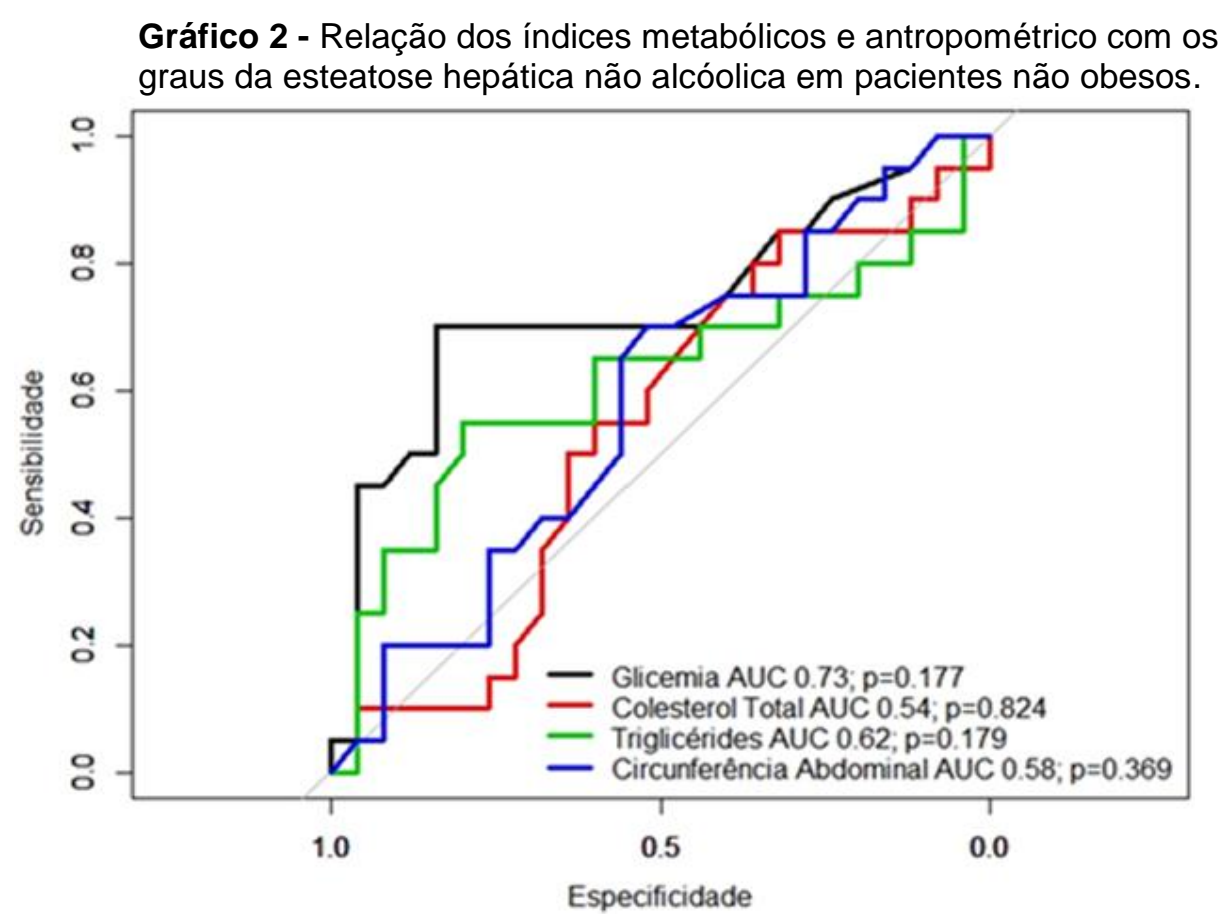

Fonte: Santana JT, et al., 2020.

\section{DISCUSSÃO}

A DHGNA foi relatada pela primeira vez por Ludwig J, et al. (1980) que descreveram seus achados histológicos em pacientes, principalmente obesos. A obesidade tem sido historicamente associada a esta patologia, porém, nem todos os pacientes obesos desenvolvem DHGNA a qual vem sendo relatada também, nos últimos anos, em pacientes não obesos. Sabe-se que a DHGNA no não obeso pode estar relacionada à predisposição genética, a componentes metabólicos e fatores dietéticos o que justificam os crescentes números nessa população (YOUSEF MH, et al., 2017).

No presente estudo, a esteatose hepática não alcóolica esteve presente em $47.1 \%$ dos pacientes obesos e 52.9\% dos pacientes não obesos. No estudo de Wei JL, et al. (2015), a prevalência da DHGNA foi de 19,3\% em pacientes não-obesos e de $60,5 \%$ em pacientes obesos. Resultados semelhantes foram encontrados nos estudos de Nabi O, et al. (2020) com um percentual de $79.1 \%$ de pacientes obesos portadores de infiltração gordura hepática e Yang MH, et al. (2016) afirmou que o predomínio de DHGNA em asiáticos não obesos variou de $11 \%$ a $21 \%$. A DHGNA, além de ser uma patologia que está fortemente associada à obesidade, tem se tornado crescente na população não obesa.

No estudo atual, a maioria dos pacientes possuía circunferência abdominal aumentada, tanto obesos quanto não obesos, com associação estaticamente significativa desse parâmetro antropométrico em relação à obesidade. Ju DY, et al. (2013) encontraram que o aumento da circunferência abdominal estava associado com um risco significativamente maior de resistência à insulina e DHGNA em adultos coreanos saudáveis.

Corroborando esse estudo, Schild BZ, et al. (2013) identificaram que a infiltração gordurosa hepática teve uma maior prevalência em pacientes que possuíram perímetro abdominal aumentado. Clemente APG, et al. (2016) reforçaram que a circunferência abdominal é um método de fácil execução e pode ser aplicada como um importante indicador antropométrico de obesidade central para a triagem de adolescentes com alto risco de DHGNA. A distribuição da adiposidade, especificamente a presença de obesidade central, é um importante fator de risco para doenças metabólicas e consequentemente para a DHGNA.

Na presente pesquisa, os exames laboratoriais tenderam a valores mais altos no grupo dos obesos e, na construção das curvas ROCs, os TG e circunferência abdominal apresentaram uma área sob curva maior que 0,7 em relação aos graus mais avançados da doença. E no que se refere aos pacientes não obesos, nenhuma das variáveis metabólicas mostrou significância estatística e apenas a glicemia de jejum apresentou uma área sob a curva ROC maior que 0.7 para os graus mais avançados de esteatose hepática. 
No estudo de Kwon YM, et al. (2012), pacientes não obesos portadores de DHGNA corresponderam a $12 \%$ de 29994 analisados e mulheres não obesas apresentaram maior taxa de hiperglicemia, hipertrigliceridemia e HDL mais baixo quando comparado à mulher obesa. Já Alam S, et al. (2014), avaliaram 229 pacientes indianos com DHGNA por histologia e constataram que os pacientes não obesos foram metabolicamente semelhantes aos obesos, sem nenhuma diferença significativa dos níveis de colesterol total, TG, HDL, glicemia de jejum e resistência à insulina. Os valores de TG e glicemia de jejum fazem parte dos critérios diagnósticos da síndrome metabólica e a incidência da DHGNA eleva-se com o aumento do número de critérios preenchidos no diagnóstico dessa síndrome.

Nesta pesquisa, a SM foi mais prevalente no grupo dos pacientes obesos do que no não obesos, apresentando associação estatisticamente significativa com a obesidade. No estudo de Leung JC, et al. (2017), foi encontrado que mais pacientes no grupo obeso foram portadores de SM comparados com o grupo não obeso, $69.8 \%$ e $43.1 \%$ respectivamente. Além disso, perceberam que os pacientes não obesos eram menos propensos a terem HAS. A DHGNA está intimamente associada aos componentes da SM, principalmente a obesidade visceral.

\section{CONCLUSÃO}

Houve associação estatisticamente significativa no grupo dos pacientes obesos portadores de esteatose hepática não alcóolica para TG e circunferência abdominal em relação a gravidade. Entretanto, não houve associação estatisticamente significativa no grupo dos pacientes não obesos portadores da esteatose hepática não alcóolica em relação às variáveis metabólicas e antropométrica analisadas. Novos estudos na população de pacientes não obesos portadores de infiltração gordurosa são necessários a fim de determinar o impacto dos fatores genéticos na patogênese dessa afecção nesse grupo.

\section{REFERÊNCIAS}

1. ADIBI A, et al. Prevalence of Nonalcoholic Fatty Liver Disease and its Related Metabolic Risk Factors in Isfahan, Iran. Adv Biomed Res, 2017;6:47.

2. ALAM S, et al. Clinical, anthropometric, biochemical, and histological characteristics of nonobese nonalcoholic fatty liver disease patients of Bangladesh. Indian J Gastroenterol, 2014; 33(5):452-7.

3. BAGHERI LK, et al. Non Alcoholic Fatty Liver Disease in Southern iran: A population based study. Hepat Mon, $2013 ; 13(5): 9248$.

4. BRAY GA, et al. Management of obesity. Lancet, 2016; 387(10031):1947-56.

5. CLEMENTE APG, et al. Circunferência da cintura como marcador para triagem de doença hepática gordurosa não alcoólica em adolescentes obesos. Revista Paulista de Pediatria, 2016; 34 (1): 47-55.

6. COTRIM HP, et al. Nonalcoholic Fatty Liver Disease Brazilian Society Of Hepatology Consensus. Arq. Gastroenterol, 2016; 53 (2): 118-122.

7. COTTER TG, RINELLA M. Nonalcoholic Fatty Liver Disease 2020: The State of the Disease. Gastroenterology, 2020;158(7):1851-1864.

8. COX DR. The regression analysis of binary sequences (with discussion). Journal of the Royal Statistical Society, 1958; 20: 215-232

9. GRAFFIGNA M, et al. Diagnóstico de esteatosis hepática por métodos clínicos, bioquímicos y por imágenes. Revista argentina de endocrinología y metabolismo,2017; 54(1), 37-46.

10. JU DY, et al. The influence of waist circumference on insulin resistance and nonalcoholic fatty liver disease in apparently healthy Korean adults. Clin Mol Hepatol, 2013;19(2):140-147.

11. KAWANO Y, COHEN DE. Mechanisms of hepatic triglyceride accumulation in non-alcoholic fatty liver disease. Journal of Gastroenterology, 2013;48(4):434-441.

12. KIM D, KIM WR. Nonobese Fatty Liver Disease. Clin Gastroenterol Hepatol, 2017;15(4):474-485.

13. KWON YM, et al. Association of nonalcoholic fatty liver disease with components of metabolic syndrome according to body mass index in Korean adults. Am J Gastroenterol , 2012; 107(12):1852-8.

14. LEUNG JC, et al. Histological severity and clinical outcomes of nonalcoholic fatty liver disease in nonobese patients. Hepatology 2017; 65:54-64.

15. LIRA NETO JCG, et al. Prevalência da síndrome metabólica e de seus componentes em pessoas com diabetes mellitus tipo 2. Texto \& Contexto - Enfermagem, 2018; 27 (3).

16. LOHMAN TG, et al. Anthropometric standardization reference manual. Champaign: Human kinetics books, 1988.

17. LUDWIG J, et al. Nonalcoholic steatohepatitis: Mayo Clinic experiences with a hitherto unnamed disease. Mayo Clin Proc. 1980; 55(7):434-438.

18. MALACHIAS MVB, et al. $7^{\text {a }}$ Diretriz Brasileira de Hipertensão Arterial: Capítulo 2 - Diagnóstico e Classificação. Arq. Bras. Cardiol., 2016; 107( 3 Suppl 3 ): 7-13 . 
19. MANN HB e WHITNEY DR. On a Test of Whether one of Two Random Variables is Stochastically Larger than the Other. The Annals of Mathematical Statistics, 1947; 18(1), 50-60.

20. MINCIS M, MINCIS R. Álcool e fígado. Gastroenterologia Endoscopia Digestiva (GED) 2011; 30(4): $152-62$.

21. NABI O, et al. Prevalence and Risk Factors of Nonalcoholic Fatty Liver Disease and Advanced Fibrosis in General Population: the French Nationwide NASH-CO Study. Gastroenterology, 2020; 159(2):791-793.

22. PEARSON K. On the Criterion that a Given System of Deviations from the Probable in the Case of a Correlated System of Variables is Such that it Can be Reasonably Supposed to have Arisen from Random Sampling. In: S. Kotz; N. L. Johnson (Eds.); Breakthroughs in Statistics: Methodology and Distribution. 1992; 50: 302, 157-175.

23. PINTÓ X, et al. A Mediterranean Diet Rich in Extra-Virgin Olive Oil Is Associated with a Reduced Prevalence of Nonalcoholic Fatty Liver Disease in Older Individuals at High Cardiovascular Risk. J Nutr, 2019; 1;149(11):1920-1929.

24. RESS C e KASER S. Mechanisms of intrahepatic triglyceride accumulation. World J Gastroenterol, 2016; 22(4): 1664-1673.

25. SAADEH SY, et al. The Utility of Radiological Imaging in Nonalcoholic Fatty Liver Disease. Gastroenterology, 2002; $123(3): 745-750$.

26. SCHILD BZ, et al. Doença hepática gordurosa não alcoólica e sua relação com a síndrome metabólica no préoperatório de pacientes submetidos à cirurgia bariátrica. Rev. Assoc. Med. Bras. 2013; 59(2):155-160.

27. SCHWABE RF, et al. Mechanisms of Fibrosis Development in Nonalcoholic Steatohepatitis. Gastroenterology, 2020;158(7):1913-1928.

28. STEFAN N, et al. Non-alcoholic fatty liver disease: causes, diagnosis, cardiometabolic consequences, and treatment strategies. Lancet Diabetes Endocrinol, 2019;7(4):313-324.

29. STUDENT. The Probable Error of a Mean. Biometrika,1908; 6(1), 1-25.

30. THE R CORE TEAM. R: A Language and Environment for Statistical Computing. Vienna, Austria: R Foundation for Statistical Computing, 2019

31. XAVIER HT, et al. V Diretriz brasileira de dislipidemias e prevenção da aterosclerose. Arquivos brasileiros de cardiologia, 2013; 101(4 Suppl 1): 1-20.

32. WEI JL, et al. Prevalence and Severity of Nonalcoholic Fatty Liver Disease in Non-Obese Patients: A Population Study Using Proton-Magnetic Resonance Spectroscopy. Am J Gastroenterol. 2015;110(9):1306-14

33. WHO. WORLD HEALTH ORGANIZATION. Physical status: the use and interpretation of anthropometry.Geneva, 1995.

34. YANG MH, et al. The associations between apolipoprotein $B, A 1$, and the B/A1 ratio and nonalcoholic fatty liver disease in both normal-weight and overweight Korean population. J Clin Lipidol, 2016;10(2):289-98.

35. YOUSEF MH, et al. Fatty liver without a large "belly": Magnified review of non-alcoholic fatty liver disease in nonobese patients. World J Gastrointest Pathophysiol, 2017;8(3):100-107. 